\title{
Practical Hadoop Security
}

Bhushan Lakhe 


\section{Practical Hadoop Security}

\section{Copyright $\odot 2014$ by Bhushan Lakhe}

This work is subject to copyright. All rights are reserved by the Publisher, whether the whole or part of the material is concerned, specifically the rights of translation, reprinting, reuse of illustrations, recitation, broadcasting, reproduction on microfilms or in any other physical way, and transmission or information storage and retrieval, electronic adaptation, computer software, or by similar or dissimilar methodology now known or hereafter developed. Exempted from this legal reservation are brief excerpts in connection with reviews or scholarly analysis or material supplied specifically for the purpose of being entered and executed on a computer system, for exclusive use by the purchaser of the work. Duplication of this publication or parts thereof is permitted only under the provisions of the Copyright Law of the Publisher's location, in its current version, and permission for use must always be obtained from Springer. Permissions for use may be obtained through RightsLink at the Copyright Clearance Center. Violations are liable to prosecution under the respective Copyright Law.

ISBN-13 (pbk): 978-1-4302-6544-3

ISBN-13 (electronic): 978-1-4302-6545-0

Trademarked names, logos, and images may appear in this book. Rather than use a trademark symbol with every occurrence of a trademarked name, logo, or image we use the names, logos, and images only in an editorial fashion and to the benefit of the trademark owner, with no intention of infringement of the trademark.

The use in this publication of trade names, trademarks, service marks, and similar terms, even if they are not identified as such, is not to be taken as an expression of opinion as to whether or not they are subject to proprietary rights.

While the advice and information in this book are believed to be true and accurate at the date of publication, neither the authors nor the editors nor the publisher can accept any legal responsibility for any errors or omissions that may be made. The publisher makes no warranty, express or implied, with respect to the material contained herein.

Managing Director: Welmoed Spahr

Acquisitions Editor: Robert Hutchinson

Developmental Editor: Linda Laflamme

Technical Reviewer: Robert L. Geiger

Editorial Board: Steve Anglin, Mark Beckner, Gary Cornell, Louise Corrigan, James DeWolf, Jonathan Gennick, Robert Hutchinson, Michelle Lowman, James Markham, Matthew Moodie, Jeff Olson, Jeffrey Pepper, Douglas Pundick, Ben Renow-Clarke, Gwenan Spearing, Matt Wade, Steve Weiss

Coordinating Editor: Rita Fernando

Copy Editor: James Fraleigh

Compositor: SPi Global

Indexer: SPi Global

Cover Designer: Anna Ishchenko

Distributed to the book trade worldwide by Springer Science+Business Media New York, 233 Spring Street, 6th Floor, New York, NY 10013. Phone 1-800-SPRINGER, fax (201) 348-4505, e-mail orders-ny@springer-sbm. com, or visit wWw. springeronline.com. Apress Media, LLC is a California LLC and the sole member (owner) is Springer Science + Business Media Finance Inc (SSBM Finance Inc). SSBM Finance Inc is a Delaware corporation.

For information on translations, please e-mail rights@apress.com, or visit wWw.apress.com.

Apress and friends of ED books may be purchased in bulk for academic, corporate, or promotional use. eBook versions and licenses are also available for most titles. For more information, reference our Special Bulk Sales-eBook Licensing web page at www. apress.com/bulk-sales.

Any source code or other supplementary material referenced by the author in this text is available to readers at WWW. apress. com. For detailed information about how to locate your book's source code, go to WWW.apress.com/source-code/. 
To my beloved father .. you will always be a part of me! 



\section{Contents at a Glance}

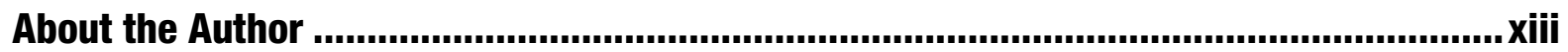

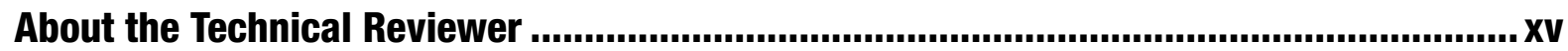

Acknowledgments ...................................................................................... xvii

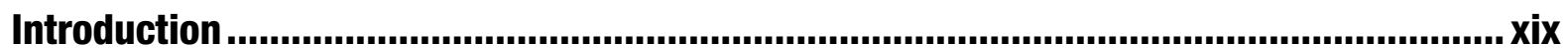

Part I: Introducing Hadoop and Its Security .......................................... 1

Chapter 1: Understanding Security Concepts ............................................................ 3

Chapter 2: Introducing Hadoop.....................................................................................19

Chapter 3: Introducing Hadoop Security ..............................................................37

Part II: Authenticating and Authorizing Within Your Hadoop Cluster ....... 49

Chapter 4: Open Source Authentication in Hadoop............................................................51

Chapter 5: Implementing Granular Authorization..............................................................75

Part IIl: Audit Logging and Security Monitoring ................................. 95

Chapter 6: Hadoop Logs: Relating and Interpretation ................................................97

Chapter 7: Monitoring in Hadoop....................................................................119

Part IV: Encryption for Hadoop .................................................... 143

Chapter 8: Encryption in Hadoop..................................................................................145 
Part V: Appendices

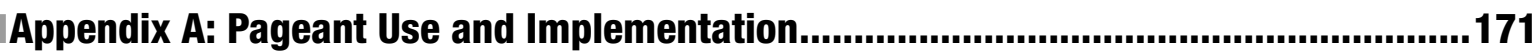

Appendix B: PuTTY and SSH Implementation for Linux-Based Clients..........................177

Appendix C: Setting Up a KeyStore and TrustStore for HTTP Encryption .........................181

Appendix D: Hadoop Metrics and Their Relevance to Security...................................183

Index 


\section{Contents}

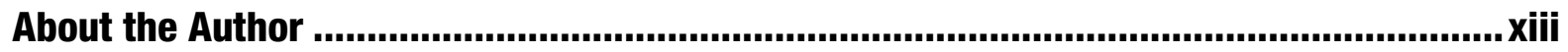

About the Technical Reviewer ......................................................................................... XV

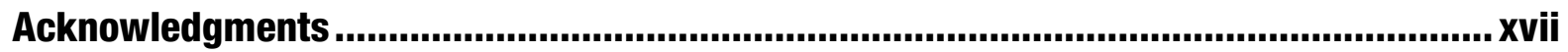

Introduction ............................................................................................................... xix

\section{Part I: Introducing Hadoop and Its Security .................................................. 1}

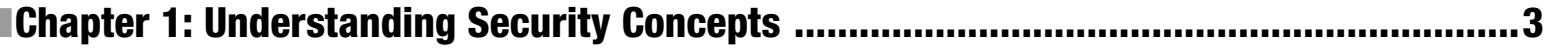

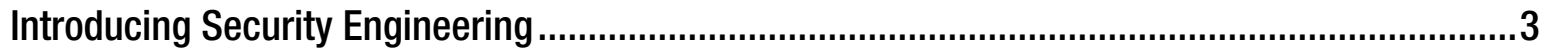

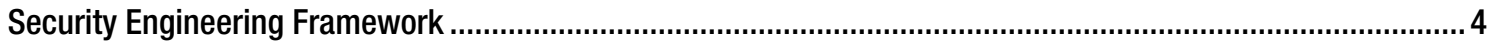

Psychological Aspects of Security Engineering ......................................................................................

Introduction to Security Protocols ...........................................................................................................

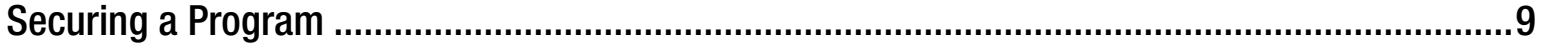

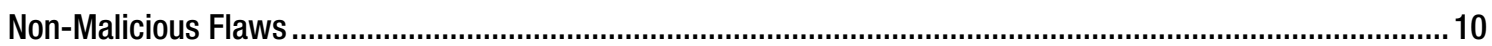

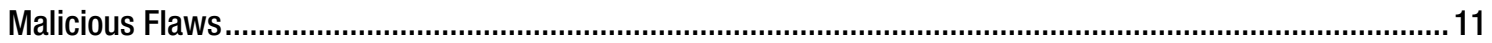

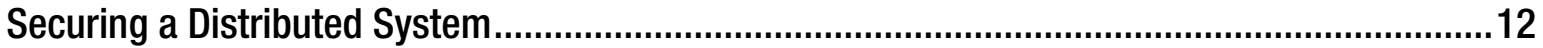

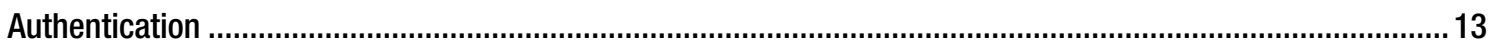

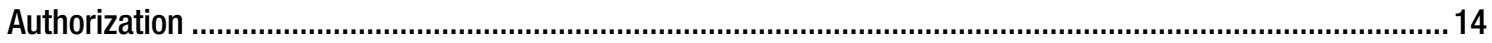

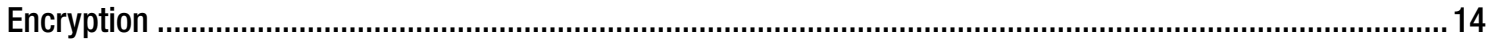

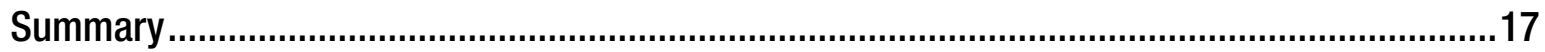

Chapter 2: Introducing Hadoop.........................................................................19

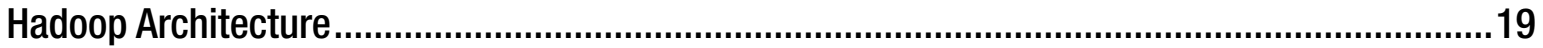

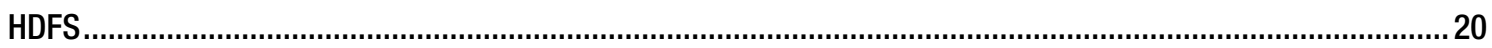

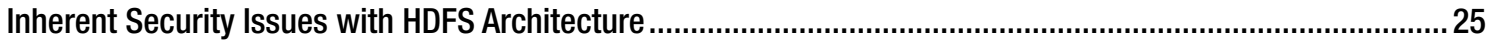




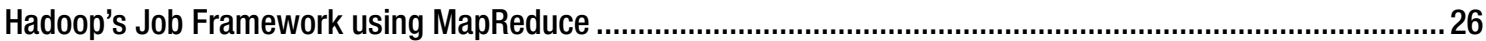

Inherent Security Issues with Hadoop's Job Framework ........................................................................... 29

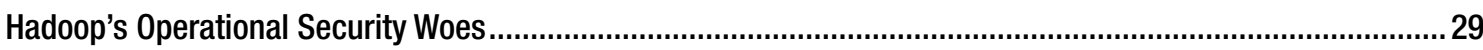

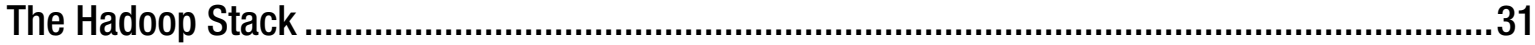

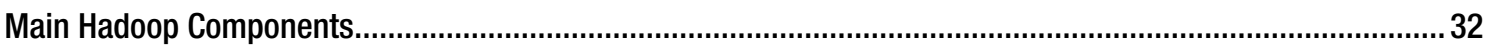

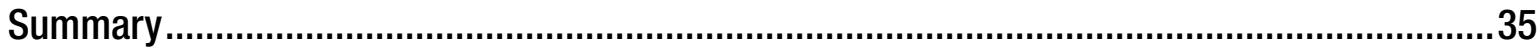

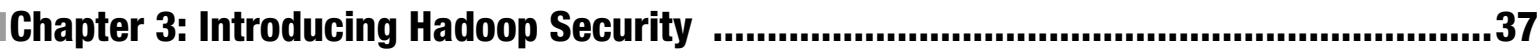

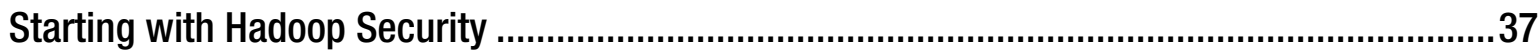

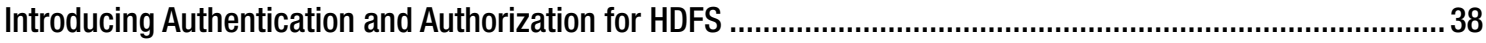

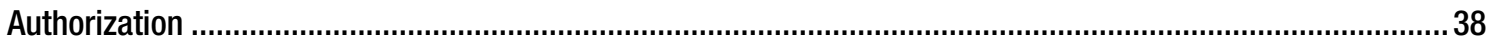

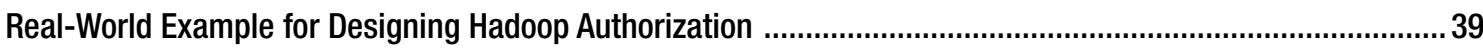

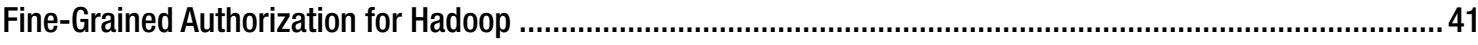

Securely Administering HDFS................................................................................. 41

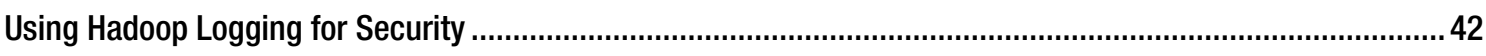

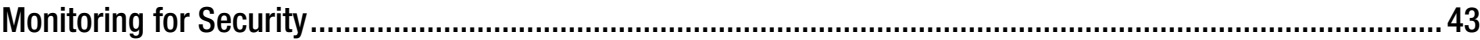

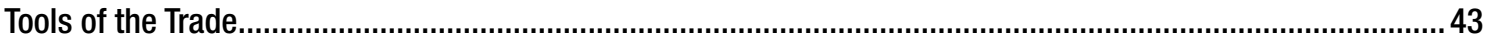

Encryption: Relevance and Implementation for Hadoop .......................................................... 45

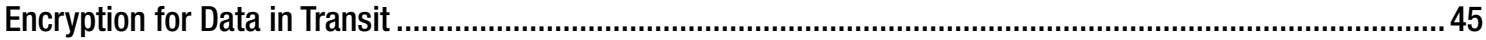

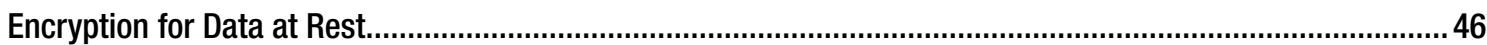

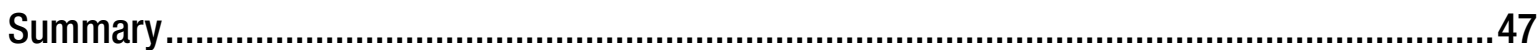

Part II: Authenticating and Authorizing Within Your Hadoop Cluster ........ 49

Chapter 4: Open Source Authentication in Hadoop......................................................51

Pieces of the Security Puzzle .........................................................................................51

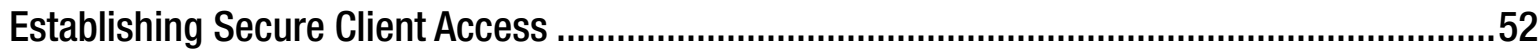

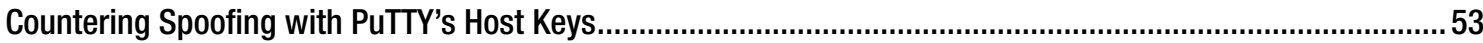

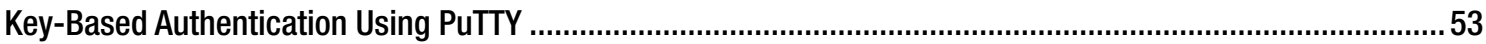

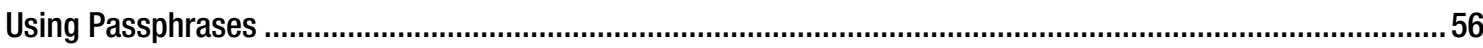




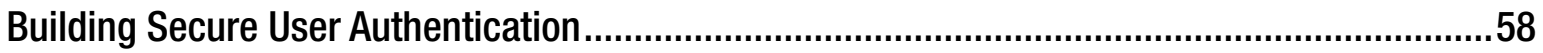

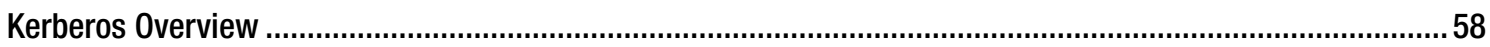

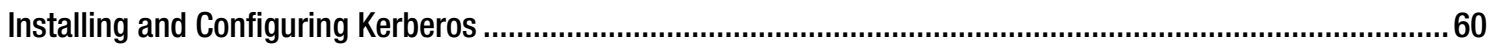

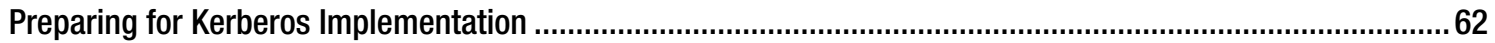

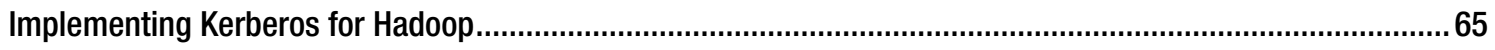

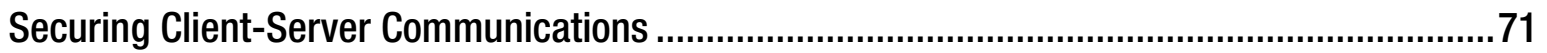

Safe Inter-process Communication ........................................................................................................ 72

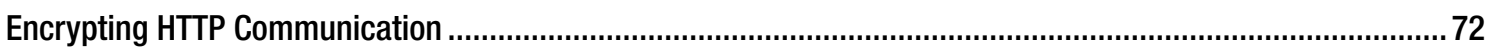

Securing Data Communication ................................................................................................................. 74

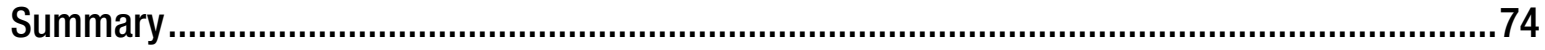

Chapter 5: Implementing Granular Authorization.................................................. 75

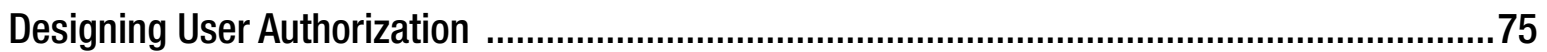

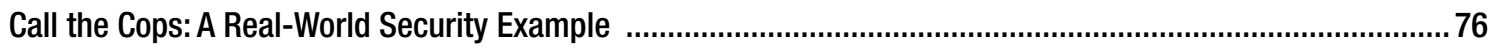

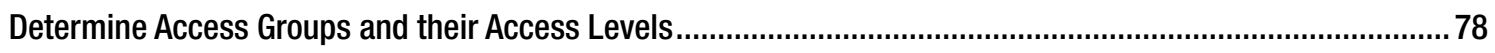

Implement the Security Model .........................................................................................................

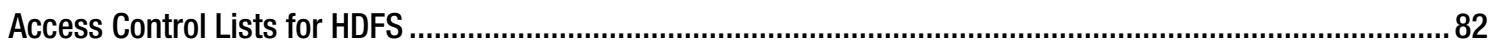

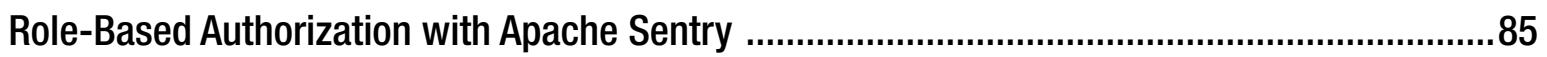

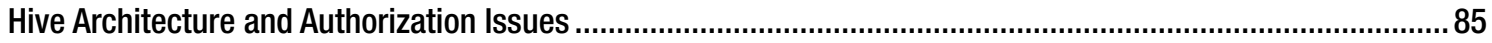

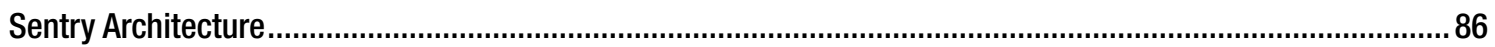

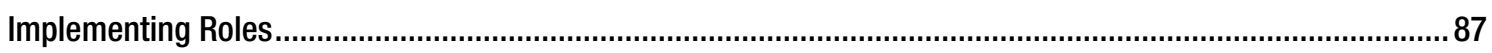

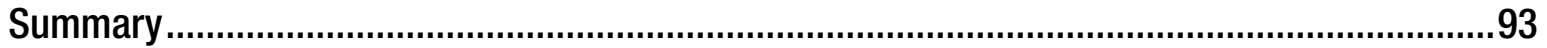

Part IIl: Audit Logging and Security Monitoring ................................ 95

Chapter 6: Hadoop Logs: Relating and Interpretation ..............................................97

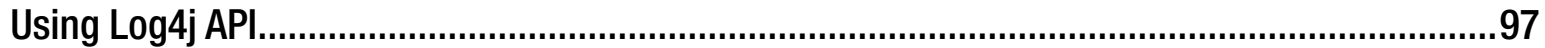

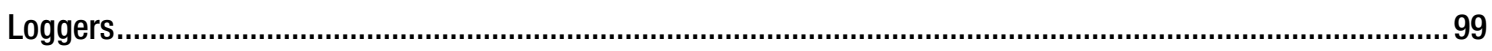

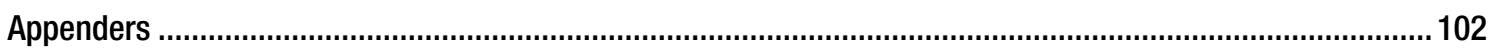

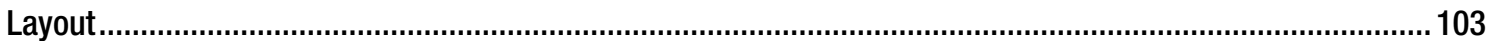

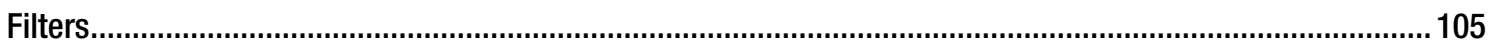


Reviewing Hadoop Audit Logs and Daemon Logs ….......................................................106

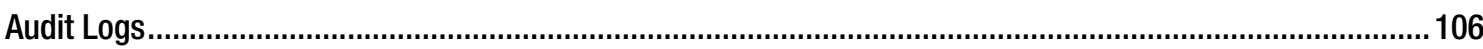

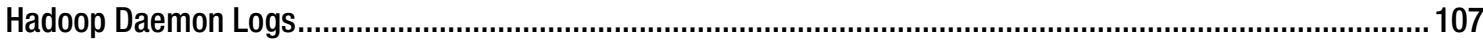

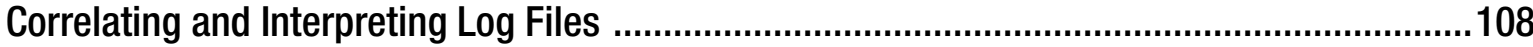

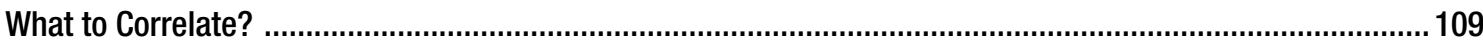

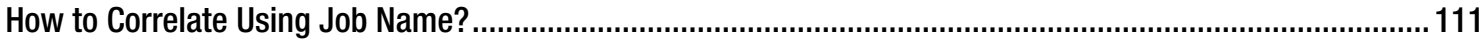

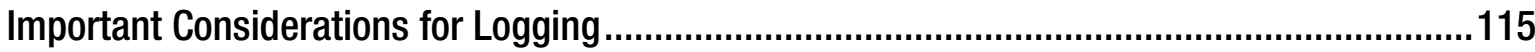

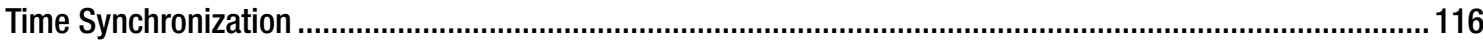

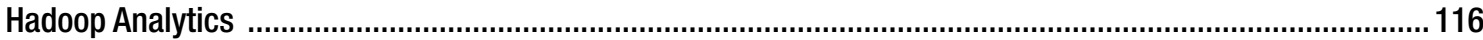

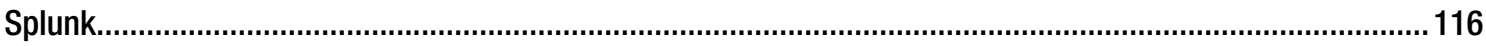

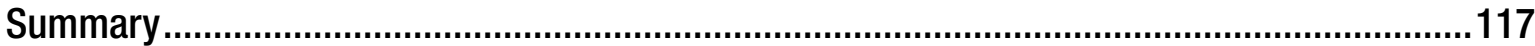

Chapter 7: Monitoring in Hadoop...............................................................................119

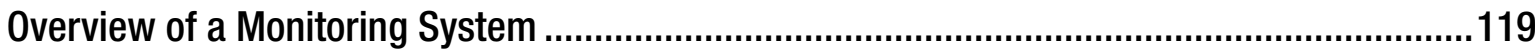

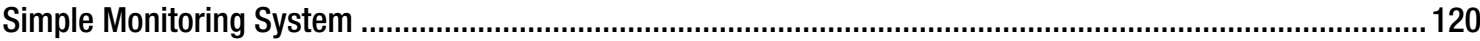

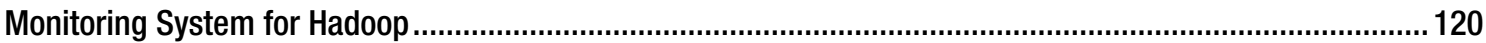

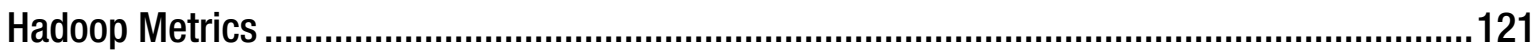

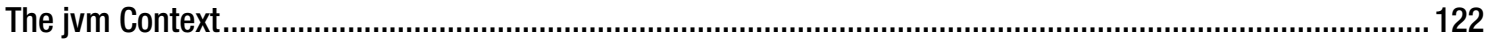

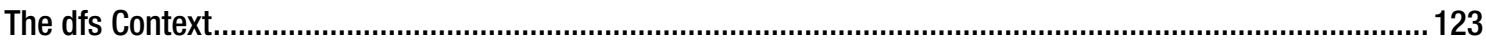

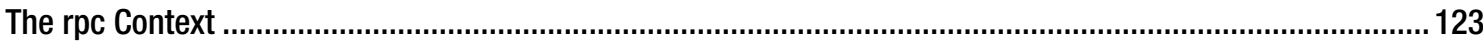

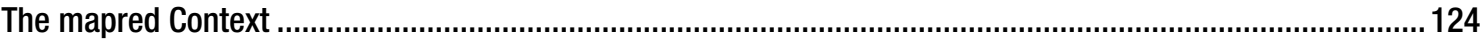

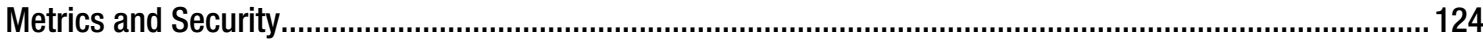

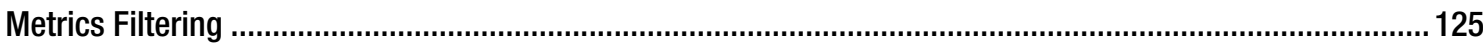

Capturing Metrics Output to File......................................................................................................126

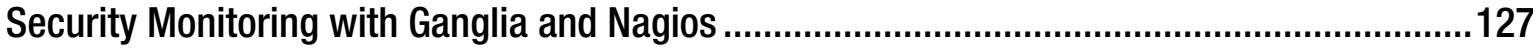

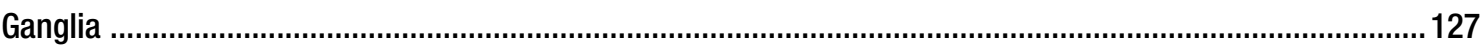

Monitoring HBase Using Ganglia ................................................................................................... 133

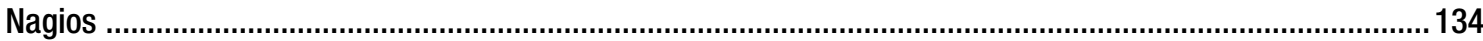

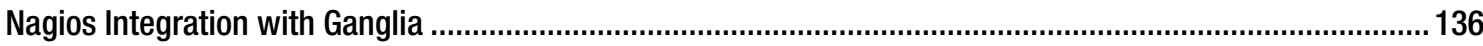

The Nagios Community....................................................................................................................141

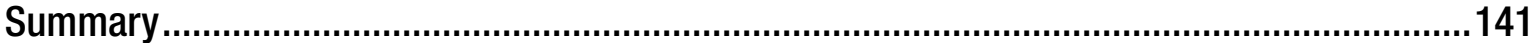


Part IV: Encryption for Hadoop

Chapter 8: Encryption in Hadoop.......................................................................145

Introduction to Data Encryption................................................................................145

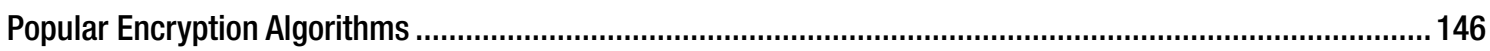

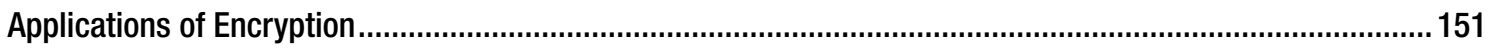

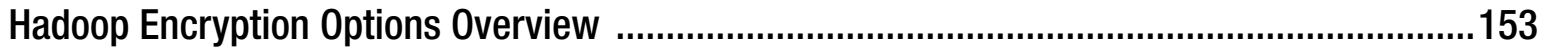

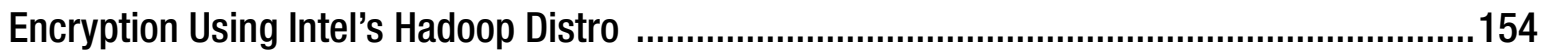

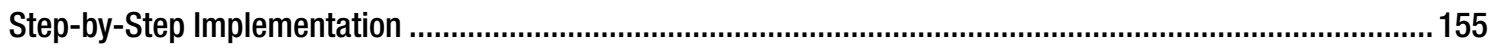

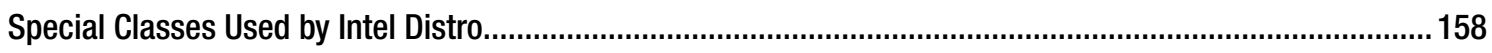

Using Amazon Web Services to Encrypt Your Data...................................................................159

Deciding on a Model for Data Encryption and Storage ......................................................................... 159

Encrypting a Data File Using Selected Model...................................................................................... 160

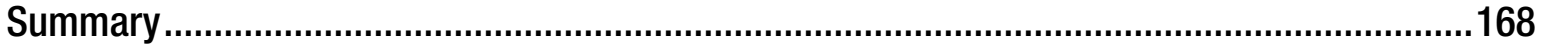

Part V: Appendices ............................................................................... 169

Appendix A: Pageant Use and Implementation.......................................................171

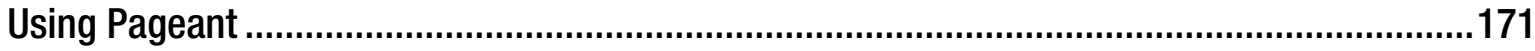

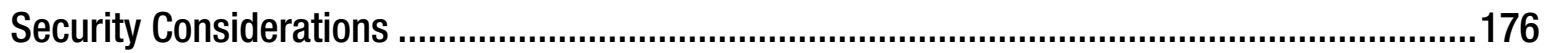

Appendix B: PuTTY and SSH Implementation for Linux-Based Clients........................177

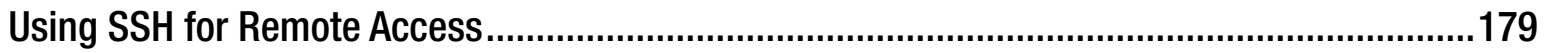

Appendix C: Setting Up a KeyStore and TrustStore for HTTP Encryption .......................181

Create HTTPS Certificates and KeyStore/TrustStore Files....................................................181

Adjust Permissions for KeyStore/TrustStore Files..............................................................182

Appendix D: Hadoop Metrics and Their Relevance to Security.....................................183

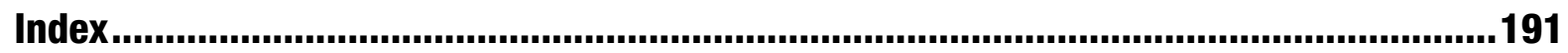





\section{About the Author}

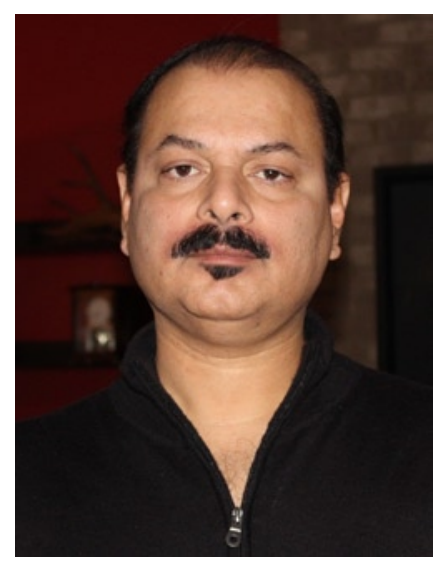

Bhushan Lakhe is a database professional, technology evangelist, and avid blogger residing in the windy city of Chicago. After graduating in 1988 from one of India's leading universities (Birla Institute of Technology \& Science, Pilani), he started his career with India's biggest software house, Tata Consultancy Services. Soon sent to the UK on a database assignment, he joined ICL, a British computer company, and worked with prestigious British clients on database assignments. Moving to Chicago in 1995, he worked as a consultant with such Fortune 50 companies as Leo Burnett, Blue Cross and Blue Shield of Illinois, CNA Insurance, ABN AMRO Bank, Abbott Laboratories, Motorola, JPMorgan Chase, and British Petroleum, often in a critical and pioneering role.

After a seven-year stint executing successful Big Data (as well as Data Warehouse) projects for IBM's clients (and receiving the company's prestigious Gerstner Award in 2012), Mr. Lakhe spent two years helping Unisys Corporation's clients with Data Warehouse and Big Data implementations. Mr. Lakhe is currently working as Senior Vice President of Information and Data Architecture at Ipsos, the world's third largest market research corporation, and is responsible for the company's Global Data Architecture and Big Data strategy. Mr. Lakhe is active in the Chicago Hadoop community and regularly answers queries on various Hadoop user forums. You can find Mr. Lakhe on LinkedIn at https://www. linkedin.com/pub/bhushanlakhe/0/455/475. 



\section{About the Technical Reviewer}

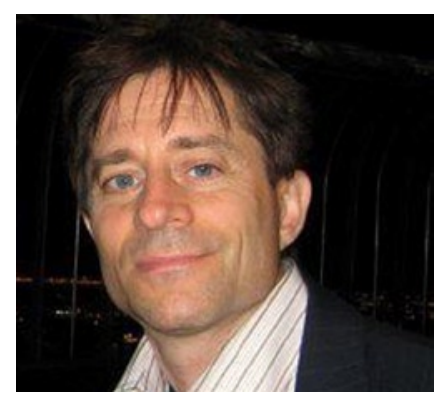

Robert L. Geiger leads product strategy for Data Driven Business Solutions and Hybrid-Transactional Processing at TransLattice, Inc. Previously, he was an architect and team lead at Pivotal, working in the areas of Big Data system security, as well as Hadoop and Big Data as a service. Mr. Geiger served as Vice President of Engineering for Mu Dynamics (formerly Mu Security). Previously, he was Senior Director of Engineering at Symantec where he led the engineering team building Symantec's award-winning SNS Intrusion Protection product, after the acquisition of Recourse Technologies. Mr. Geiger spent 10 years at Motorola Labs working on electromagnetic systems modeling using massively parallel supercomputers, wireless data systems development, mobile security software, and e-commerce solutions. He holds several patents in the areas of mobile data, wireless security, and e-commerce. Mr Geiger has a Masters of Electrical Engineering degree from the University of Illinois, Urbana, and a Bachelor of Science degree in Electrical Engineering from the State University of New York. 



\section{Acknowledgments}

While writing this book, I received help (both directly and indirectly) from a lot of people, and I would like to thank them all. Thanks to the Hadoop community and the user forums, from whom I have learned a great deal. There are many selfless people in the Hadoop community, and I feel that's the biggest strength of the "Hadoop revolution." In particular, thanks to my friend Satya Kondapalli for introducing Hadoop to me, and to my friends Naveed Asem and Zafar Ahmed for keeping me motivated!

I would like to thank the Intel Technical team (Bala Subramanian, Sunjay Karan, Manjunatha Prabhu) in Chicago for their help with Intel's Hadoop distribution and encryption at rest, as well as Cloudera's Justin Kestelyn for providing information about Sentry. Last, I would like to thank my friend Milind Bhandarkar (of Pivotal) for his help and support.

I am grateful to my editors, Linda Laflamme and Rita Fernando, at Apress for their help in getting this book together. Linda and Rita have been there throughout to answer any questions that I have, to read and improve my first (and second and third ...) drafts, and to keep me on schedule! I am also very thankful to Robert Geiger for taking time to review my book technically. Bob always had great suggestions for improving a topic, recommended additional details, and, of course, resolved technical shortcomings!

Last, writing this book has been a lot of work, and I couldn't have done it without the constant support from my family. My wife, Swati, and my kids, Anish and Riya, have been very understanding. I'm looking forward to spending lots more time with all of them! 



\section{Introduction}

Last year, I was designing security for a client who was looking for a reference book that talked about security implementations in the Hadoop arena, simply so he could avoid known issues and pitfalls. To my chagrin, I couldn't locate a single book for him that covered the security aspect of Hadoop in detail or provided options for people who were planning to secure their clusters holding sensitive data! I was disappointed and surprised. Everyone planning to secure their Hadoop cluster must have been going through similar frustration. So I decided to put my security design experience to broader use and write the book myself.

As Hadoop gains more corporate support and usage by the day, we all need to recognize and focus on the security aspects of Hadoop. Corporate implementations also involve following regulations and laws for data protection and confidentiality, and such security issues are a driving force for making Hadoop "corporation ready."

Open-source software usually lacks organized documentation and consensus on performing a particular functional task uniquely, and Hadoop is no different in that regard. The various distributions that mushroomed in last few years vary in their implementation of various Hadoop functions, and some, such as authorization or encryption, are not even provided by all the vendor distributions. So, in this way, Hadoop is like Unix of the '80s or '90s: Open source development has led to a large number of variations and in some cases deviations from functionality. Because of these variations, devising a common strategy to secure your Hadoop installation is difficult. In this book, I have tried to provide a strategy and solution (an open source solution when possible) that will apply in most of the cases, but exceptions may exist, especially if you use a Hadoop distribution that's not well-known.

It's been a great and exciting journey developing this book, and I deliberately say "developing," because I believe that authoring a technical book is very similar to working on a software project. There are challenges, rewards, exciting developments, and of course, unforeseen obstacles—not to mention deadlines!

\section{Who This Book Is For}

This book is an excellent resource for IT managers planning a production Hadoop environment or Hadoop administrators who want to secure their environment. This book is also for Hadoop developers who wish to implement security in their environments, as well as students who wish to learn about Hadoop security. This book assumes a basic understanding of Hadoop (although the first chapter revisits many basic concepts), Kerberos, relational databases, and Hive, plus an intermediate-level understanding of Linux.

\section{How This Book Is Structured}

The book is divided in five parts: Part I, "Introducing Hadoop and Its Security," contains Chapters 1, 2, and 3; Part II, "Authenticating and Authorizing Within Your Hadoop Cluster," spans Chapters 4 and 5; Part III, "Audit Logging and Security Monitoring," houses Chapters 6 and 7; Part IV, "Encryption for Hadoop," contains Chapter 8; and Part V holds the four appendices. 
Here's a preview of each chapter in more detail:

- Chapter 1, "Understanding Security Concepts," offers an overview of security, the security engineering framework, security protocols (including Kerberos), and possible security attacks. This chapter also explains how to secure a distributed system and discusses Microsoft SQL Server as an example of secure system.

- Chapter 2, "Introducing Hadoop," introduces the Hadoop architecture and Hadoop Distributed File System (HDFS), and explains the security issues inherent to HDFS and why it's easy to break into a HDFS installation. It also introduces Hadoop's MapReduce framework and discusses its security shortcomings. Last, it discusses the Hadoop Stack.

- Chapter 3, "Introducing Hadoop Security," serves as a roadmap to techniques for designing and implementing security for Hadoop. It introduces authentication (using Kerberos) for providing secure access, authorization to specify the level of access, and monitoring for unauthorized access or unforeseen malicious attacks (using tools like Ganglia or Nagios). You'll also learn the importance of logging all access to Hadoop daemons (using the Log4j logging system) and importance of data encryption (both in transit and at rest).

- Chapter 4, "Open Source Authentication in Hadoop," discusses how to secure your Hadoop cluster using open source solutions. It starts by securing a client using PuTTY, then describes the Kerberos architecture and details a Kerberos implementation for Hadoop step by step. In addition, you'll learn how to secure interprocess communication that uses the RPC (remote procedure call) protocol, how to encrypt HTTP communication, and how to secure the data communication that uses DTP (data transfer protocol).

- Chapter 5, "Implementing Granular Authorization," starts with ways to determine security needs (based on application) and then examines methods to design fine-grained authorization for applications. Directory- and file-level permissions are demonstrated using a real-world example, and then the same example is re-implemented using HDFS Access Control Lists and Apache Sentry with Hive.

- Chapter 6, "Hadoop Logs: Relating and Interpretation," discusses the use of logging for security. After a high-level discussion of the Log4j API and how to use it for audit logging, the chapter examines the Log4j logging levels and their purposes. You'll learn how to correlate Hadoop logs to implement security effectively, get a look at Hadoop analytics and a possible implementation using Splunk.

- Chapter 7, "Monitoring in Hadoop," discusses monitoring for security. It starts by discussing features that a monitoring system needs, with an emphasis on monitoring distributed clusters. Thereafter, it discusses the Hadoop metrics you can use for security purposes and examines the use of Ganglia and Nagios, the two most popular monitoring applications for Hadoop. It concludes by discussing some helpful plug-ins for Ganglia and Nagios that provide securityrelated functionality and also discusses Ganglia integration with Nagios.

- Chapter 8, "Encryption in Hadoop," begins with some data encryption basics, discusses popular encryption algorithms and their applications (certificates, keys, hash functions, digital signatures), defines what can be encrypted for a Hadoop cluster, and lists some of the popular vendor options for encryption. A detailed implementation of HDFS and Hive data at rest follows, showing Intel's distribution in action. The chapter concludes with a step-by-step implementation of encryption at rest using Elastic MapReduce VM (EMR) from Amazon Web Services. 


\section{Downloading the Code}

The source code for this book is available in ZIP file format in the Downloads section of the Apress web site (wWw.apress.com).

\section{Contacting the Author}

You can reach Bhushan Lakhe at blakhe@aol.com or bclakhe@gmail.com. 